\title{
Nanotecnologia: uma prospecção tecnológica no âmbito nacional e internacional
}

\author{
Nanotechnology: a technological prospection in the national and \\ international environment
}

\author{
Diego Fialkoski ${ }^{1}$ \\ Carlos Ricardo Maneck Malfatti ${ }^{1}$ \\ ${ }^{1}$ Universidade Estadual do Centro-Oeste, Guarapuava, PR, Brasil
}

\begin{abstract}
Resumo
A Nanotecnologia é a tecnologia que estuda a matéria em nanoescala, em escala atômica e molecular. Essa tecnologia é empregada na modernização de setores industriais e tecnológicos como a tecnologia da informação, energia, meio ambiente, segurança, tecnologia de alimentos, transporte e até na medicina. O estudo prospectivo analisou o avanço das pesquisas em nanotecnologia, precisamente nas publicações de artigos e nos depósitos de patentes, no período de 1997 até 2017. As bases de dados pesquisadas foram o Periódico CAPES para artigos e o INPI e USPTO para depósito de patentes. Constatou-se uma grande quantidade de artigos e apenas 7 patentes no Brasil, enquanto que na base de patentes americanas encontraram-se 79 patentes nos últimos 20 anos. O estudo prospectivo demonstra que nos Estados Unidos o número dos indicadores de pesquisa e desenvolvimento da tecnologia, bem como a sua transformação em produto, é maior.
\end{abstract}

Palavras-chave: Nanotecnologia. Patente. Prospecção Tecnológica.

\begin{abstract}
Nanotechnology is the technology which study substance at nanoscale, atomic and molecular scale. This technology is used in the modernization of industrial and technological sectors such as information technology, energy, environment, safety, food technology, transportation and even in medicine. The prospective study analyzed the progress of nanotechnology research, articles publications and patent deposits between 1997 to 2017. The databases searched were the CAPES Journal for articles, INPI and USPTO for patent filed. A large number of Articles was found and only 7 patents in Brazil, while in the American patent database 79 patents in the last 20 years. The prospective study shows that the indicators of research and development of technology as well as its transformation into product is bigger in the United States.
\end{abstract}

Keywords: Nanotechnology. Patent. Technological Prospecting.

Área tecnológica: Nanotecnologia.

\section{Introdução}

Para Longo (2004), a Nanotecnologia é o termo utilizado para descrever a criação, manipulação e exploração de materiais com escala nanométrica. A unidade de medida do nanômetro, $\mathrm{nm}$, é um metro dividido por um bilhão, ou seja, $10^{-9} \mathrm{~m}$. No estudo da Agência Brasileira de Desenvolvimento Industrial (ABDI) (2010), vários países como EUA, Japão, países da Europa e 
o Brasil perceberam que a nanotecnologia representa um novo patamar de conhecimento, com visão para imensos impactos científicos e econômicos. Estes países vêm tomando iniciativas que poderão render significativas melhorias na qualidade de vida de suas populações com o avanço em diversos setores, como, por exemplo, da agricultura, energia, preservação ambiental, saúde e outros. Nos últimos anos, o Brasil tem avançado consistentemente no desenvolvimento de ações de muita importância em Ciência, Tecnologia e Inovação (CT\&I), com resultados concretos na produção científica, tecnológica e formação de recursos humanos em áreas consideradas estratégicas, particularmente em determinados campos de nanotecnologia (ABDI, 2010, p. 92) .

Segundo Canalli e Silva (2012), atualmente é indiscutível a importância de patentes e sua direta ligação com o desenvolvimento da tecnologia e da sociedade. O sistema de patentes de proteção às invenções, às marcas e aos direitos autorais evolui desde seus primórdios.

Ferreira, Guimarães e Contador (2009) destacam que dentre as vantagens oferecidas pelas patentes pode-se citar, além dos incentivos ao desenvolvimento tecnológico, o encorajamento à pesquisa científica, a disseminação do conhecimento prático e econômico, a criação de novos mercados e a satisfação das necessidades latentes dos consumidores.

O Estudo Prospectivo realizado visa a fornecer informações sobre o avanço na produção de artigos e patentes referente às pesquisas em nanotecnologia.

\section{Revisão da Literatura}

\subsection{Nanotecnologia}

O físico Richard Feynman profere uma palestra na Sociedade Americana de Física, na Califórnia, e esse discurso é considerado como o marco inicial da nanotecnologia (FEYNMAN, 1960). Na palestra intitulada "Há muito espaço na parte inferior", do inglês There's plenty of room at the bottom, Feynman (Prêmio Nobel de Física de 1965) instigou os espectadores sobre a possibilidade de escrever a Enciclopédia Britânica na cabeça de um alfinete. Mesmo não sabendo exatamente como fazer, ele sabia que poderia manipular individualmente os átomos.

Para Rey (2003), a nanotecnologia é considerada multidisciplinar e envolve profissionais como químicos, físicos, biomédicos, engenheiros, farmacêuticos, entre outros. Mas são os químicos os principais responsáveis pelo desenvolvimento da nanotecnologia. A Química é a ciência que trata da composição, estrutura e propriedades da matéria, em nível atômico e molecular, assim como as reações entre os elementos ou as moléculas. Químicos dos colóides trabalhavam com nanomateriais desde o final do século XIX.

Schober (2002) afirma que o desenvolvimento das indústrias automobilística, eletrônica, farmacêutica e têxtil, entre tantas outras, tem uma grande contribuição por parte da Nanotecnologia. A língua eletrônica é um resultado brasileiro, desenvolvida pela Empresa Brasileira de Pesquisa Agropecuária (Embrapa), com parceria da Escola Politécnica da Universidade de São Paulo (USP) e da USP de São Carlos, mediante o Instituto de Ciências Matemáticas e de Computação e o Instituto de Física. A invenção é capaz de detectar por meio de sensores, com agilidade e precisão, padrões do paladar como amargo, azedo, doce e salgado, com potencial aplicação na indústria de bebidas, para análise de café, cerveja, vinho etc.

De acordo com Service (2007), os Estados Unidos dispõem em seu mercado de aproximadamente 380 produtos com nanotecnologia. 


\subsection{Estudo Prospectivo}

Segundo Antunes (2002), desde a década de 50, o termo Prospecção Tecnológica deu-se a partir de registros iniciais de utilização sistematizada das informações como ferramenta estratégica, sendo o objetivo principal a redução do tempo entre a invenção e a disposição dos novos produtos no mercado. A partir da década de 80 a prospecção passou a ganhar mais força na utilização dos métodos, acelerando o desenvolvimento tecnológico da atualidade.

Para Para Pelaez e Semrecsabyi (2006), estudos prospectivos indicam algumas situações nas quais se aplicam com mais frequência, como: apoio ao planejamento de programas de pesquisa na direção de propor investimentos e instituir a capacitação cientifica necessários; ajuda no programa de desenvolvimento tecnológico pretendendo aceitação de uma nova tecnologia ou adaptação a novas demandas tecnológicas; reconhecimento de áreas de atividade tecnológica as quais serão importantes ao aprimoramento ou ao início de novos produtos; instituição de modelo de produtividade e qualidade de novos produtos, novos processos ou novos materiais; avaliação do período de introdução de nova tecnologia no mercado; reconhecimento do potencial econômico de uma nova tecnologia; planejamento tecnológico de longo prazo; identificação de novas oportunidades tecnológicas; identificação de impactos sociais de uma nova tecnologia; reconhecimento de novas formas políticas em consequência da incorporação de novas tecnologias pela sociedade.

Kupfer e Tigre (2004) classificam os métodos de Prospecção Tecnológica em três grupos:

a) Monitoramento (Assessment), que consiste no acompanhamento sistemático e contínuo da progressão dos fatos e na identificação de fatores portadores de mudança;

b) Previsão (Forecasting), que consiste na realização de projeções baseadas em informações históricas e modelagem de tendências;

c) Visão (Foresight), que é a antecipação de possibilidades futuras, com base em interação não estruturada entre especialistas.

\section{Metodologia}

O estudo utiliza a pesquisa bibliográfica para fundamentação teórica e bases de artigos e patentes como ferramenta de prospecção, com a finalidade de fazer um monitoramento dos pedidos de patentes relacionadas à Nanotecnologia usando as plataformas brasileiras e americana. No trabalho foram utilizados dois indicadores.

\subsection{Artigos - CAPES}

O primeiro indicador foi a coleta de dados no estágio da pesquisa, utilizando-se a base de dados da CAPES no período de 1997 até 2017.

$\mathrm{Na}$ pesquisa realizada neste trabalho foram utilizadas a seguinte palavra-chave: "nanotecnologia" e a palavra-chave em inglês: "nanotechnology".

A pesquisa foi realizada no site: <www.capes.gov.br $>$, conforme o fluxograma da Figura 1. 
Figura 1 - Fluxograma da busca de periódicos na CAPES

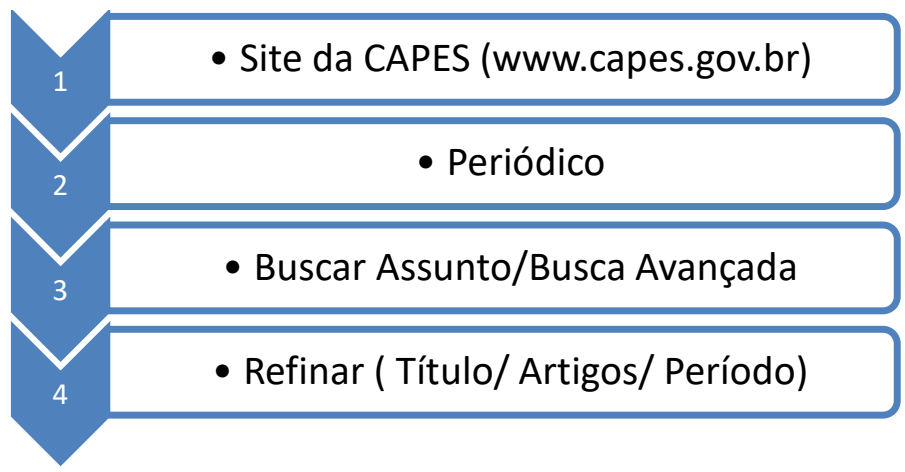

Fonte: Elaborada pelos autores deste artigo (2018)

\subsection{Patentes - INPI/USPTO}

O segundo indicador foi o desenvolvimento com números de patentes em Nanotecnologia. A busca foi realizada em duas etapas: a primeira foi a escolha das bases de dados a serem utilizadas como fonte de pesquisa de documento de patente; e a segunda foi pela estratégia de busca da patente.

Em um primeiro momento foi escolhida a base de dados de patentes disponível no Instituto Nacional de Propriedade Industrial (INPI), que apresentou todos os documentos depositados no Brasil. Outra base de dados foi a do Escritório Americano United States Patent and Trademark Office (USPTO). As bases de dados utilizadas disponibilizam dados bibliográficos dos pedidos de patentes depositados no Brasil e nos Estados Unidos.

Após a escolha da base de dados de patentes, a segunda etapa realizada foi a busca de patentes na base de dados. Foi feito um levantamento do número total de depósitos de patentes no período de 1976 a 2016. Em um primeiro momento foi feita a busca no INPI apenas com o termo "nanotecnologia" no título. Em um segundo momento, repetiu-se a mesma estratégia de busca de patentes na base de dados USPTO.

A pesquisa foi realizada no INPI ( <www.inpi.gov.br>), conforme o fluxograma da Figura 2.

Figura 2 - Fluxograma da Base de Patentes da Plataforma do INPI

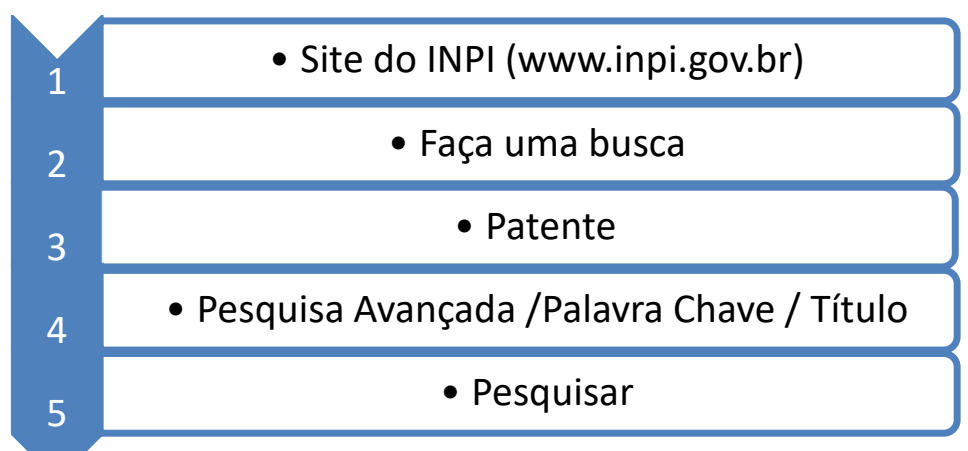

Fonte: Elaborada pelos autores deste artigo (2018)

A pesquisa foi realizada no USPTO (<www.uspto.gov>), conforme o fluxograma da Figura 3. 
Figura 3 - Fluxograma da Base de Patentes da Plataforma do USPTO

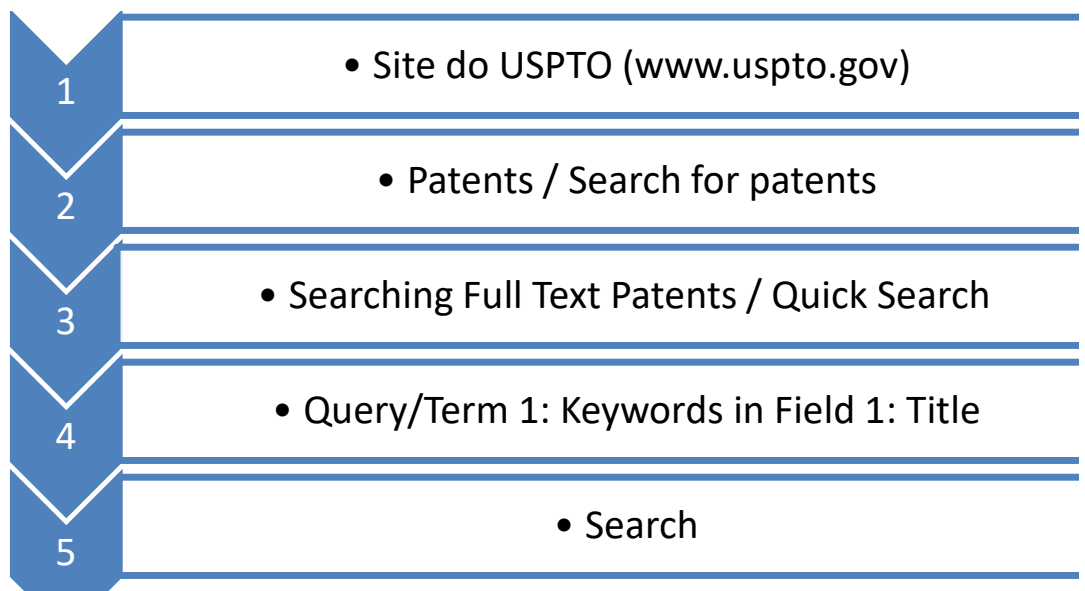

Fonte: Elaborada pelos autores deste artigo (2018)

Após a pesquisa utilizaram-se as ferramentas de prospecção tecnológica com o objetivo de prospectar visões futuras, utilizando-se alguns métodos de prospecção como: monitoramento (Assessment); previsão (Forecasting); e visão (Foresight).

\section{Resultados e Discussão}

\subsection{Indicador 1: pesquisa de artigos científicos}

Na Figura 4 são apresentados os números totais de artigos encontrados na base de dados da CAPES.

Figura 4 - Artigos publicados na base de dados da CAPES para as palavras-chave "nanotecnologia" e "nanotechnology", no período de 1997-2017

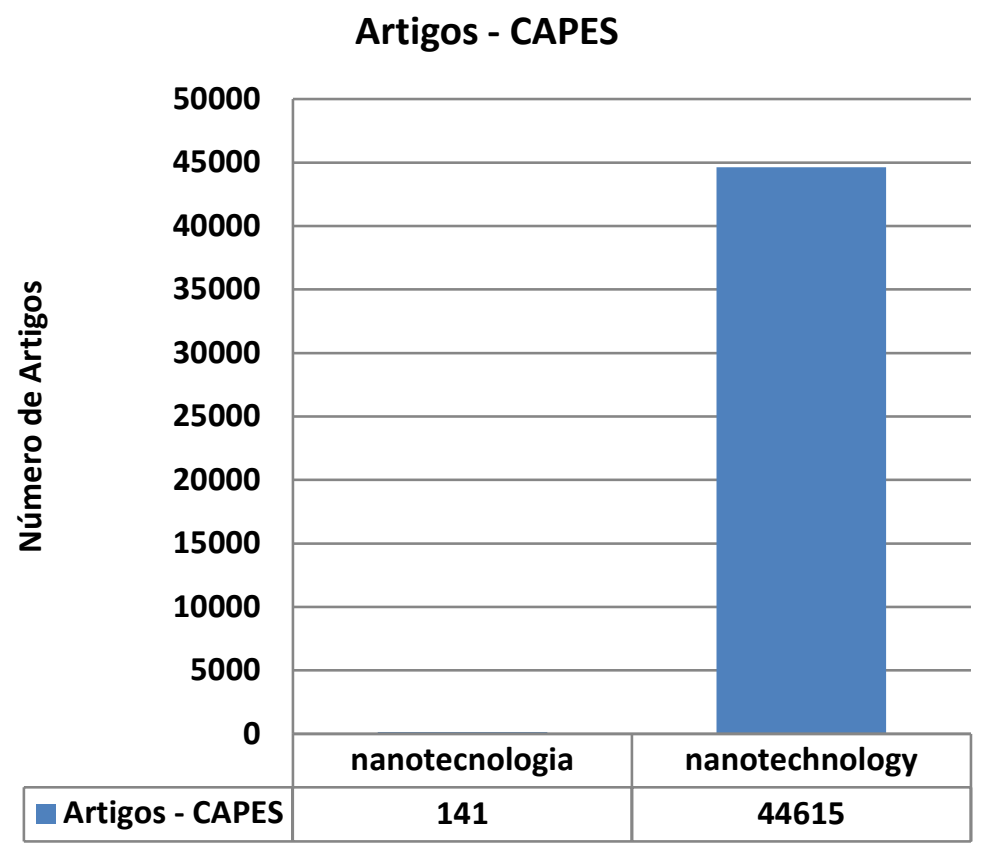

Fonte: Elaborada pelos autores deste artigo (2018) 
Com a palavra-chave em português "nanotecnologia" encontraram-se 141 artigos. Com a palavra-chave em inglês "nanotechnology" o resultado foi de 44.615 artigos. Como se vê na Figura 4, foi possível identificar uma superioridade em número de artigos em inglês em comparação com os artigos em português. A explicação é pelo fato de a língua inglesa ser mais abrangente, considerada como linguagem universal, gerando, assim, uma maior visibilidade.

Na Figura 5 são apresentados os anos de publicação de artigos disponíveis na base de dados da CAPES com as palavras-chave em português e inglês.

Figura 5 - Ano de publicação dos artigos na base de dados da CAPES

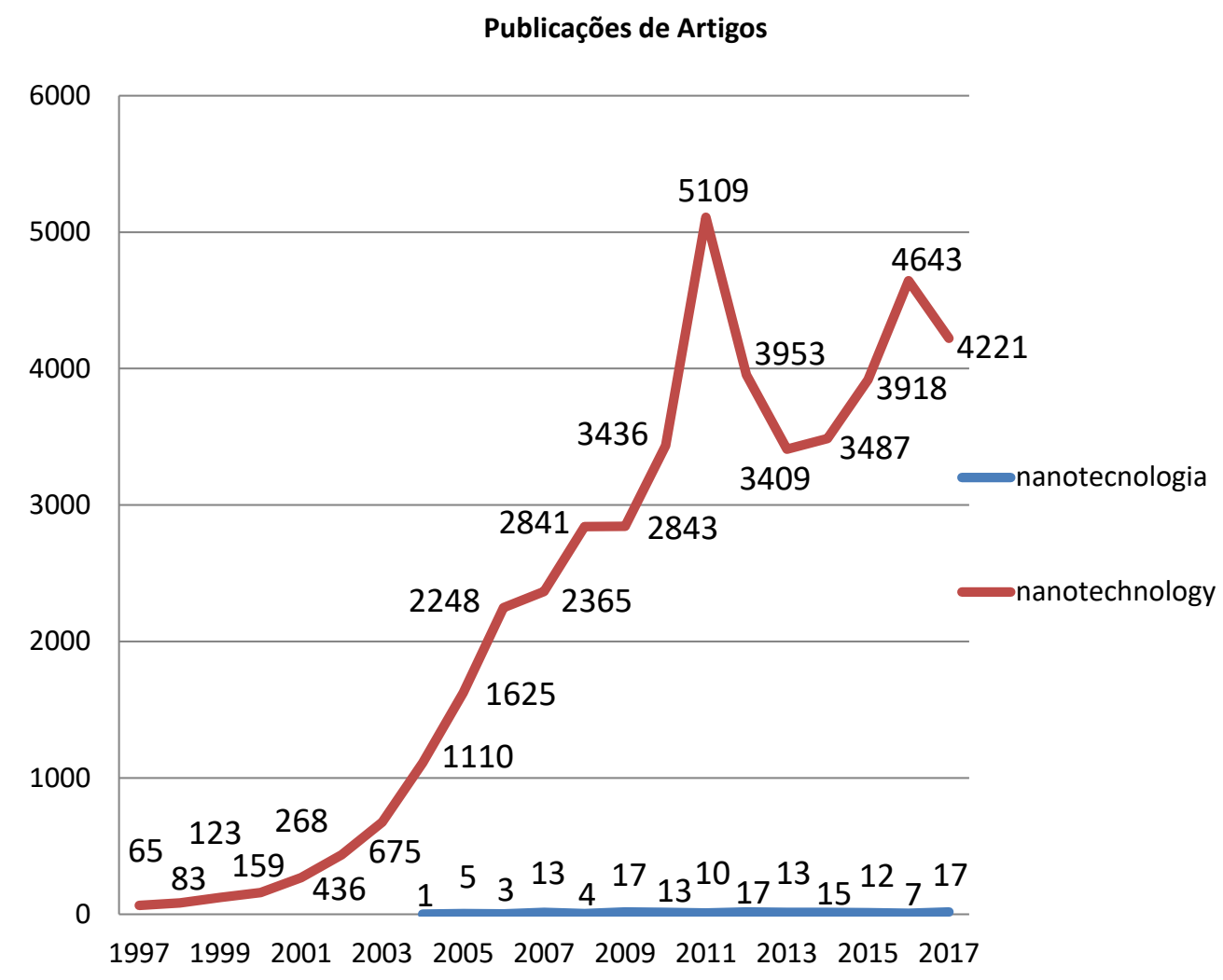

Fonte: Elaborada pelos autores deste artigo (2018)

Verifica-se na Figura 5 a evolução das publicações, sendo consideravelmente maior na língua inglesa como previsto: foi possível observar um crescimento exponencial até 2011, chegando a 5.109 publicações naquele ano. No caso dos artigos em português encontraram-se poucas publicações: nos anos mais produtivos houve apenas 17 publicações.

\subsection{Indicador 2: pesquisas de patentes}

A busca de patentes depositadas com relação à Nanotecnologia, com a coleta de dados do indicador de desenvolvimento de números de patentes, foi realizada nas bases: INPI (BR) $e$ USPTO (EUA), onde estão disponíveis os resultados.

Na Figura 6 são apresentados os resultados de patentes depositadas no Brasil, com dados disponíveis no Instituto Nacional de Propriedade Intelectual (INPI). 
Figura 6 - Patentes na base de dados do Instituto Nacional de Propriedade Intelectual (INPI), no período de 2003 a 2016

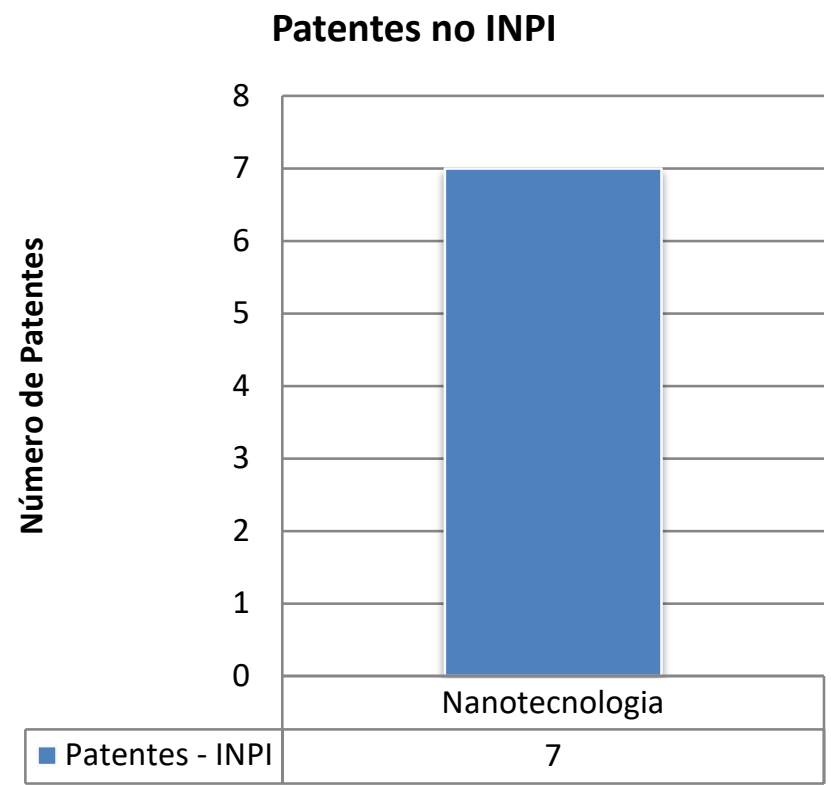

Fonte: Elaborada pelos autores deste artigo (2018)

Com a análise constataram-se sete depósitos, sendo seis patentes de invenção e um modelo de utilidade (MU), um quantitativo de aproximadamente $5 \%$ em relação aos artigos publicados em português.

Os dados apresentados na Figura 7 são patentes depositadas nos EUA, encontradas no banco de dados do USPTO.

Figura 7 - Patentes na base de dados do USPTO, no período de 2000 a 2015

\section{Patentes no USPTO}

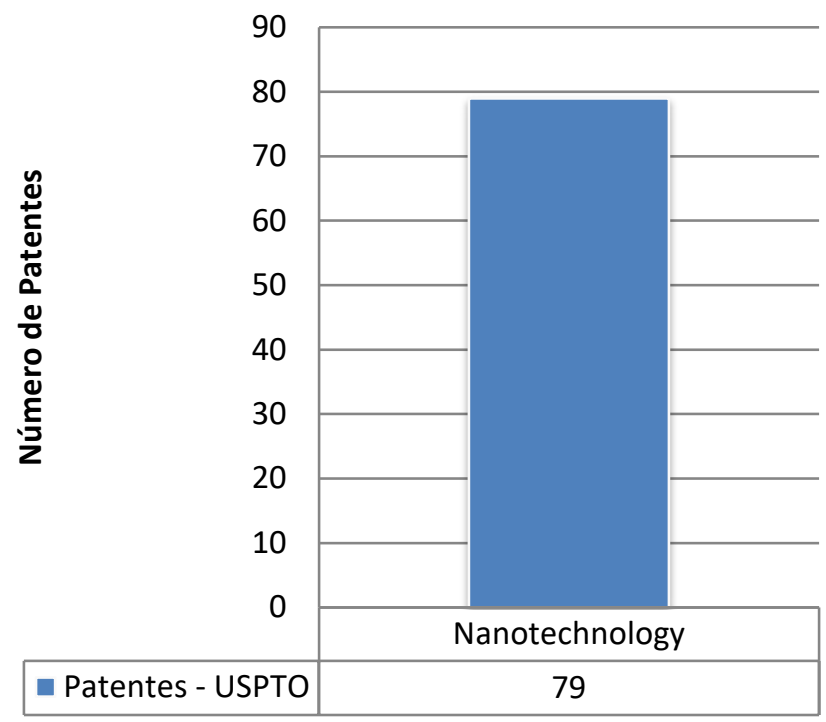

Fonte: Elaborada pelos autores deste artigo (2018) 
Verifica-se na Figura 7 que, referente à busca com a palavra-chave "nanotechnology", foi possível observar uma quantidade de 79 patentes. Esse valor significa dez vezes mais que a quantidade de depósitos no banco de dados do INPI.

\subsection{Pedidos de Patente de Nanotecnologia Depositados no Brasil}

A Figura 8 demonstra o desenvolvimento do patenteamento em Nanotecnologia no Brasil, a partir da primeira patente, que foi depositada no ano de 2003.

Figura 8 - Ano de publicação de pedidos patentes na base de dados do INPI

\section{Patentes no INPI}

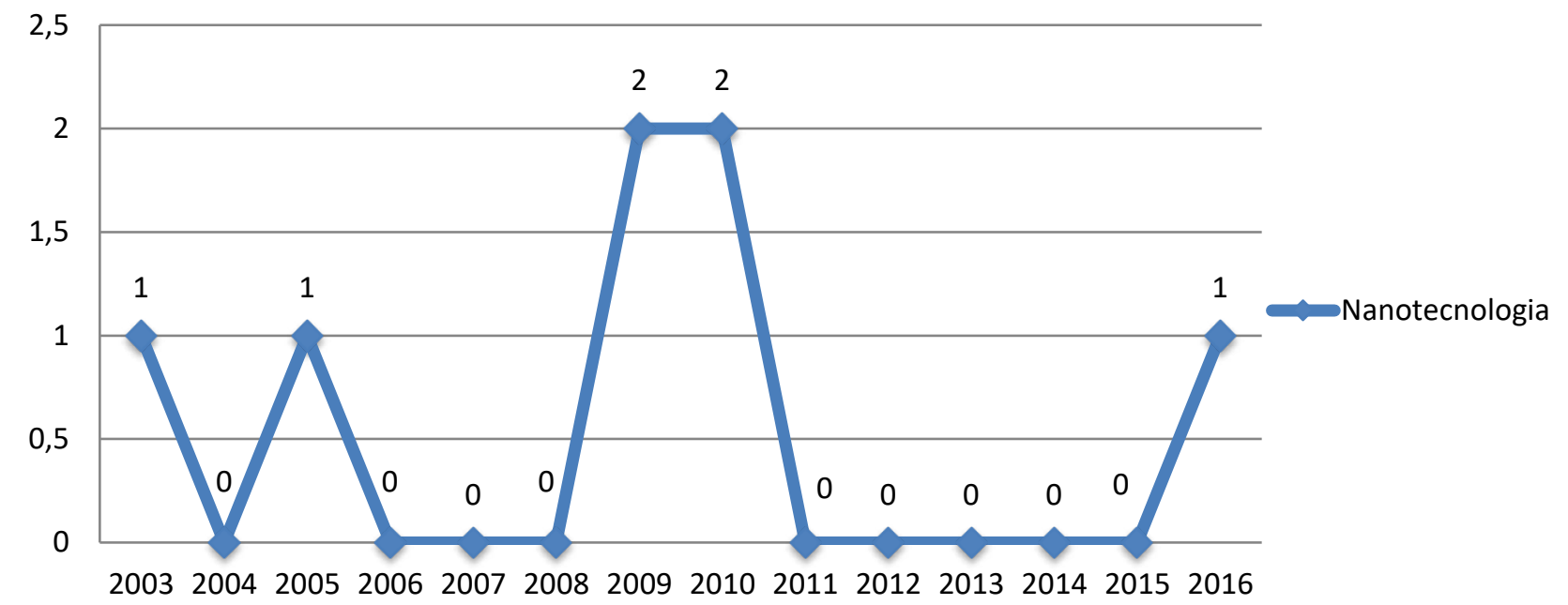

Fonte: Elaborada pelos autores deste artigo (2018)

Com seis depósitos de patentes e um modelo de utilidade (MU), pode-se constatar um valor baixo de depósito, com isso impossibilitando um estudo prospectivo nas patentes do INPI.

Na Figura 9 são apresentados os países de origem dos depositantes, no Brasil, de pedidos de patente referente à nanotecnologia publicados no período de 2003-2016.

Figura 9 - Países depositantes na base de dados do INPI

\section{Países Depositantes}

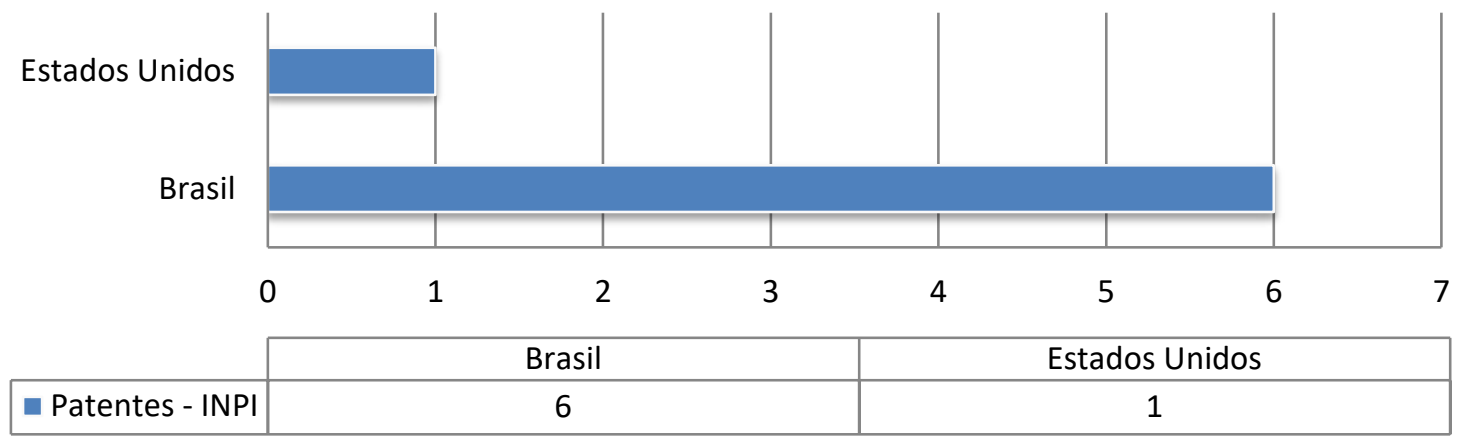

Número de Patentes

Fonte: Elaborada pelos autores deste artigo (2018) 
Com a análise da Figura 9, encontraram-se seis depósitos feitos por brasileiros e um por americanos.

\subsection{Pedidos de Patente em Nanotecnologia Depositados nos Estados Unidos}

O levantamento realizado na base de dados USPTO, obteve o resultado de 79 pedidos de patente relacionados à nanotecnologia nos Estados Unidos, no período entre 2000 e 2015.

A Figura 10 demonstra o desenvolvimento dos pedidos de patenteamento referentes à nanotecnologia nos Estados Unidos.

Figura 10 - Ano de pedido de patentes na base de dados do USPTO

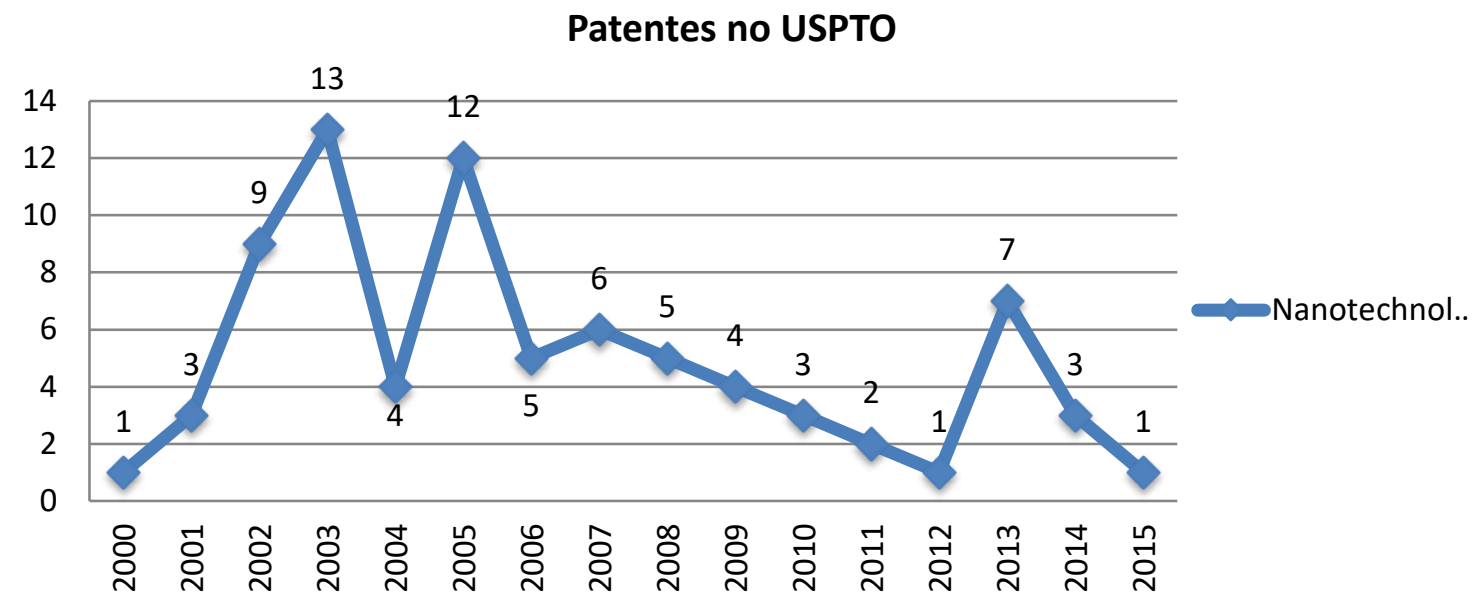

Fonte: Elaborada pelos autores deste artigo (2018)

A Figura 11 mostra os países depositantes na base de dados do USPTO.

Figura 11 - Países depositantes na base de dados do USPTO

Patentes - USPTO

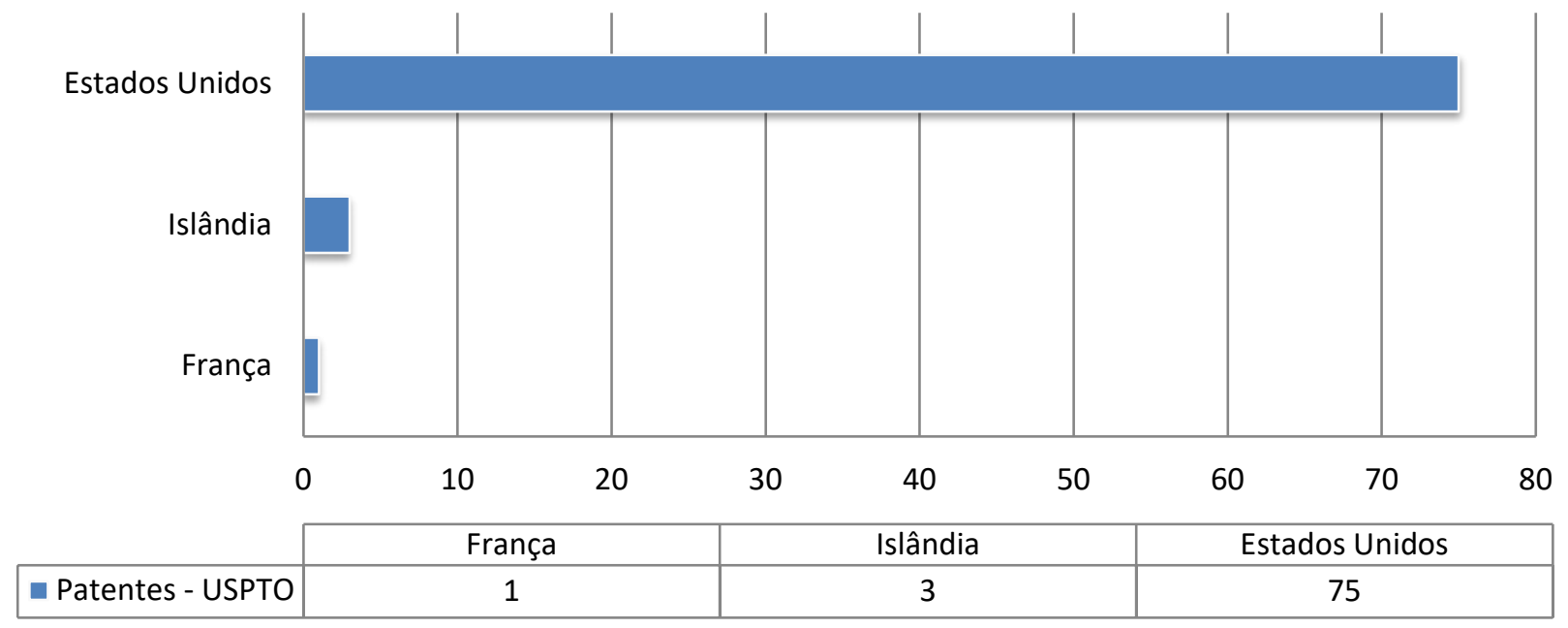

Fonte: Elaborada pelos autores deste artigo (2018) 
Na Figura 11, observam-se os depósitos feitos por franceses, islandeses e americanos, com predominância de depósitos dos Estados Unidos.

\subsection{Análise de Patenteamento Referente à Nanotecnologia na Base de Dados do INPI e do USPTO}

A Figura 12 demonstra o desenvolvimento do patenteamento em Nanotecnologia no INPI e no USPTO.

Figura 12 - Número de depósitos de patentes na base de dados no INPI e no USPTO

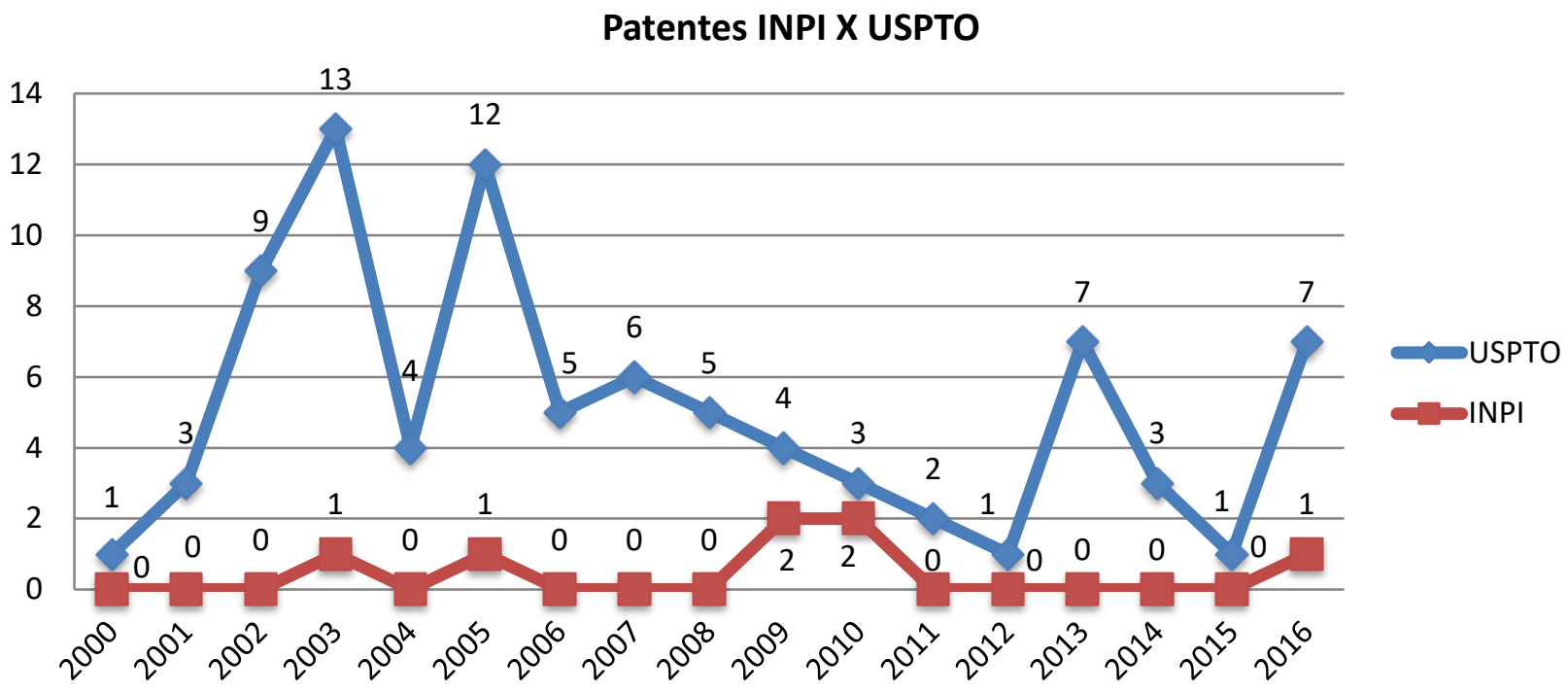

Fonte: Elaborada pelos autores deste artigo (2018)

No gráfico da Figura 12, percebe-se que os depósitos de patentes em nanotecnologia começaram a partir de 2000 nos Estados Unidos e em 2003 no Brasil. O número de depósito em patente nos Estados Unidos é 10 vezes maior que no Brasil.

\section{Considerações Finais}

Com a pesquisa desenvolvida encontraram-se 44.756 artigos sobre nanotecnologia no banco de dados da CAPES nos últimos 20 anos. No INPI encontraram-se apenas seis pedidos de patentes de invenção e um modelo de utilidade e no USPTO 79 pedidos no mesmo período. Constatou-se que os Estados Unidos é o principal responsável pelas pesquisas em nanotecnologia, já que o país desenvolve muitos produtos eletrônicos, farmacêuticos, médicos entre outros que fazem uso dessa tecnologia. Outro motivo também se deve ao fato do alto investimento que se necessita em laboratórios, microscópios avançados para as análises das matérias em escala nanométrica. É nítido que o número de artigos é superior ao número de registros de patentes. Pela quantidade elevada de artigos publicados, pode-se concluir que existe uma grande quantidade de pesquisas no escopo nanotecnologia e que não repercutem proporcionalmente em inovações patenteáveis. 


\section{Referências}

ABDI - Agência Brasileira de Desenvolvimento Industrial. Estudo Prospectivo Nanotecnologia. Brasília, DF: Agência Brasileira de Desenvolvimento Industrial, 2010. p. 92. (Série Cadernos da indústria ABDI XX). ISBN 978-85-61323-23-3.

CANALLI, W. M.; SILVA, R. P. Uma breve história das patentes: analogias entre ciência x tecnologia e trabalho intelectual x trabalho operacional. In: Anais eletrônicos do CONGRESSO SCIENTIARUM HISTORIA V, 2012, Rio de Janeiro: Universidade Federal do Rio de Janeiro(UFRJ), 2012. v. 11, p. 742-748. Disponível em:<http://www.hcte.ufrj.br/downloads/sh/sh4/trabalhos/ Waldemar\%2520Canalli.pdf > . Acesso em: 20 jun. 2018. ISSN 2176-1248.

FERREIRA, A. A.; GUIMARÃES, E. R.; CONTADOR, J. C. Patente como instrumento competitivo e fonte de informação tecnológica. Revista Gestão \& Produção, v. 16, n. 2, p. 209-221, 2009. ISSN 0104-530X.

FEYNMAN, R. P. Há mais espaços lá embaixo. Pasadena, CA: Caltech's Engineering and Science. Pasadena, 1960.

KUPFER, D.; TIGRE, P. Prospecção Tecnológica. Em: Modelo SENAI de prospecção: Documento Metodológico. s.l.: Montevideo, 2004.

LONGO, E. Nanotecnologia. Anais eletrônicos da 56ª REUNIÃO ANUAL DA SBPC, 07, 2004, Cuiabá: Universidade Federal do Mato Grosso (UFMT), 2004. Disponível em:<http://www.sbpcnet.org. br/livro/56ra/banco_conf_simp/textos/ElsonLongo.htm>. Acesso em 20 jun. 2018.

PELAEZ, V.; SEMRECSABYI, T. (Org.). Economia da Inovação Tecnológica. São Paulo: Hucitec, 2006.

REY, L. Dicionário de Termos Técnicos de Medicina e Saúde. 2. ed. Rio de Janeiro: Guanabara Koogan, 2003.

SANTOS, M. M.; COELHO, G. M.; SANTOS, D. M.; FILHO, L. F. Prospecção de tecnologias de futuro: métodos, técnicas e abordagens. Parcerias Estratégicas, n. 19, 2004.

SCHOBER J. Nanotecnologia ainda não está no dia a dia das pessoas. Revista Virtual ComCiência, v. 37, 2002.

SERVICE, R. F. Health and Safety Research Slated for Sizable Gains. Revista Science, v. 315, p. 926, 2007.

\section{Sobre os autores}

\section{Diego Fialkoski}

E-mail: diegodiscovery@gmail.com

Graduado em Matemática (Licenciatura Plena) pela Universidade Estadual do Centro-Oeste UNICENTRO (2010); e em Logística pelo Centro Universitário UNICESUMAR (2015). Pós-graduado em Educação Matemática pela UNIVALE (2011). Mestrando em Propriedade Intelectual e Transferência de Tecnologia para a Inovação-PROFNIT pela Universidade Estadual do Centro-Oeste (UNICENTRO).

Endereço Profissional: Agência de Inovação Tecnológica de Guarapuava (NOVATEC). Rua Simeão Camargo Varela de Sá, 03, Vila Carli. Campus Universitário CEDETEG. CEP: 85040-080. Guarapuava-PR. 


\section{Carlos Ricardo Maneck Malfatti}

E-mail: crmalfatti@gmail.com

Graduado em Biomedicina (Habilitado em Análises Clínicas e Imagem) e em Educação Física (Licenciatura Plena). Mestre em Ciências Biológicas: Bioquímica, pela Universidade Federal de Santa Maria UFSM (2001). Doutor em

Ciências Biológicas: Bioquímica, pela Universidade Federal do Rio Grande do Sul (UFRGS) (2007). Pós-Doutor em Tecnologia de Processos Químicos e Bioquímicos, pela UTFPR (2014).

Endereço Profissional: Agência de Inovação Tecnológica de Guarapuava (NOVATEC). Rua Simeão Camargo Varela de Sá, 03, Vila Carli. Campus Universitário CEDETEG. CEP: 85040-080. Guarapuava-PR. 